\title{
On subexponential tails for the maxima of negatively driven compound renewal and Lévy processes
}

\author{
Dmitry Korshunov \\ Lancaster University, UK. E-mail: d.korshunov@lancaster.ac.uk
}

\begin{abstract}
We study subexponential tail asymptotics for the distribution of the maximum $M_{t}:=\sup _{u \in[0, t]} X_{u}$ of a process $X_{t}$ with negative drift for the entire range of $t>0$. We consider compound renewal processes with linear drift and Lévy processes. For both processes we also formulate and prove the principle of a single big jump for their maxima. The class of compound renewal processes with drift particularly includes the Cramér-Lundberg renewal risk process.
\end{abstract}

Keywords: Lévy process, compound renewal process, distribution tails, heavy tails, long-tailed distributions, subexponential distributions, random walk

2000 MSC: 60F10, 60G51, 60K05

\section{Introduction}

For a probability distribution $F$ on the real line, let $F(x)=F(-\infty, x]$ denote the distribution function and $\bar{F}(x)=F(x, \infty)=1-F(x)$ its tail. We say that $F$ is (right-) heavy-tailed distribution if all its positive exponential moments are infinite, $\int_{\mathbb{R}} e^{s x} F(d x)=\infty$ for all $s>0$. Otherwise we call $F$ (right-) light-tailed.

In the presence of heavy tails, the class $\mathcal{S}$ of subexponential distributions is of basic importance. A distribution $F$ on $\mathbb{R}^{+}$with unbounded support is called subexponential if $\overline{F * F}(x) \sim 2 \bar{F}(x)$ as $x \rightarrow \infty$. Any subexponential distribution is known (see, e.g., Foss et al. (2013, Lemma 3.2)) to be longtailed, i.e., for all fixed $y, \bar{F}(x+y) \sim \bar{F}(x)$ as $x \rightarrow \infty$.

A distribution $F$ of a random variable $Y$ valued on the whole real line is called subexponential if the distribution $F^{+}$of $Y^{+}$is so.

The class of subexponential distributions plays an important role in many applications, for instance, for waiting times in the $G I / G / 1$ queue 
and for ruin probabilities - see, e.g., Asmussen (2003, Ch. X.9); Asmussen and Albrecher (2010, Ch. X); Embrechts et al. (1997, Sec. 1.4); Rolski et al. (1998).

A distribution $F$ on $\mathbb{R}$ with right unbounded support and finite mean is called strong subexponential $\left(F \in \mathcal{S}^{*}\right)$ if

$$
\int_{0}^{x} \bar{F}(x-y) \bar{F}(y) d y \sim 2 \bar{F}(x) \int_{0}^{\infty} \bar{F}(y) d y \quad \text { as } x \rightarrow \infty .
$$

It is known - see, e.g., Foss et al. (2013, Theorem 3.27) — that $F \in \mathcal{S}^{*}$ implies both $F \in \mathcal{S}$ and $F_{I} \in \mathcal{S}$ where $F_{I}$ is the integrated tail distribution defined by its tail,

$$
\bar{F}_{I}(x):=\min \left(1, \int_{x}^{\infty} \bar{F}(y) d y\right), \quad x>0 .
$$

Let $Y, Y_{1}, Y_{2}, \ldots$ be i.i.d. random variables with a negative expectation $b=\mathbb{E} Y<0$. Consider a random walk $S_{0}=0, S_{n}=Y_{1}+\ldots+Y_{n}$ and its maximum

$$
M_{n}^{S}:=\max _{0 \leq k \leq n} \sum_{i=1}^{k} Y_{i}
$$

hereinafter we follow the standard convention $\sum_{i=1}^{0} f(i)=0$.

Since $b<0$, the family $M_{n}^{S}, n \geq 1$, is stochastically bounded. Let $B$ be the distribution of $Y_{1}^{+}$and $B_{I}$ be the integrated tail distribution of $Y_{1}^{+}$. As well known for the overall maximum of the random walk,

$$
M_{\infty}^{S}=\max _{n \geq 0} \sum_{i=1}^{n} Y_{i}
$$

the asymptotic relation

$$
\mathbb{P}\left\{M_{\infty}^{S}>x\right\} \sim \bar{B}_{I}(x) /|b| \quad \text { as } x \rightarrow \infty
$$

holds in the heavy-tailed case if and only if the integrated tail distribution $B_{I}$ is subexponential - see e.g. Theorem 5.12 in Foss et al. (2013). Also, if $B$ is strong subexponential, $B \in \mathcal{S}^{*}$, then the following tail result holds for finite time horizon maxima

$$
\mathbb{P}\left\{\max _{k \leq n} \sum_{i=1}^{k} Y_{i}>x\right\} \sim \frac{1}{|b|} \int_{x}^{x+n|b|} \bar{B}(v) d v
$$


as $x \rightarrow \infty$ uniformly for all $n \geq 1$ - see Korshunov (2002) or Foss et al. (2013, Theorem 5.3); uniformity for all $n \geq 1$ means that

$$
\sup _{n \geq 1}\left|\frac{\mathbb{P}\left\{\max _{k \leq n} \sum_{i=1}^{k} Y_{i}>x\right\}}{\frac{1}{|b|} \int_{x}^{x+n|b|} \bar{B}(v) d v}-1\right| \rightarrow 0 \quad \text { as } x \rightarrow \infty .
$$

So the subexponential tail behaviour for the maxima of random walks is well understood while surprisingly much less is known for Lévy processes. In this contribution we particularly demonstrate in Section 2 how results for random walks relate to those for the compound renewal process with linear drift in the presence of heavy-tails - see Theorem 1; in particular, we formulate and prove the principle of a single big jump in Theorem 4. Based on that we give in Section 3 a very general treatment of subexponential tail behaviour for Lévy processes with negative drift-see Theorem 5 . In Section 4 we derive tail asymptotics for a Lévy process stopped at random time and for its maximum within this time interval. An application to the Cramér-Lundberg renewal risk model is given in Section 5. A discussion of results available in the literature may be found just after Theorems 1 and 5 .

\section{Asymptotics for compound renewal processes with linear drift}

Consider a compound renewal process with linear drift $X_{t}$ which is defined as

$$
X_{t}=\sum_{i=1}^{N_{t}} Y_{i}+c t
$$

where $c$ is some real constant, $N_{t}$ is a renewal process generated by jump epochs $0=T_{0}<T_{1}<T_{2}<\ldots$, where $\tau_{n}:=T_{n}-T_{n-1}>0$ are i.i.d. random variables with finite mean $\mathbb{E} \tau=: 1 / \lambda$, and $Y_{n}, n \geq 1$, are i.i.d. jumps with finite mean $b$. The $Y_{n}$ 's are supposed to be independent of the process $N_{t}$.

We assume that the drift of the process is negative, that is, $a:=c+b \lambda<$ 0 , hence the family of distributions of maxima

$$
M_{t}:=\max _{u \in[0, t]} X_{u}
$$

is tight,

$$
\sup _{t>0} \mathbb{P}\left\{M_{t}>x\right\} \leq \mathbb{P}\left\{M_{\infty}>x\right\} \rightarrow 0 \quad \text { as } x \rightarrow \infty .
$$

We are interested in the tail behaviour of $M_{t}$. 
If $c<0$ and $Y_{1} \geq 0$-which corresponds to the Cramér-Lundberg renewal model - then the overall maximum $M_{\infty}$ is simply the maximum of the associated random walk:

$$
M_{\infty}=\max _{n \geq 0} \sum_{i=1}^{n}\left(Y_{i}+c \tau_{i}\right),
$$

because the maximum of $X_{t}$ can only occur at a jump epoch. Let $B$ be the distribution of $Y_{1}^{+}$and $B_{I}$ be the integrated tail distribution. Since

$$
\mathbb{P}\left\{Y_{1}>x+h\right\} \mathbb{P}\left\{c \tau_{1}>-h\right\} \leq \mathbb{P}\left\{Y_{1}+c \tau_{1}>x\right\} \leq \mathbb{P}\left\{Y_{1}>x\right\}
$$

for $c<0$ and all $h>0$, the integrated tail distribution of $Y_{1}+c \tau_{1}$ is asymptotically equivalent to $\bar{B}_{I}(x)$ provided $B_{I}$ is a long-tailed distribution. Then it follows from (1) that the equivalence

$$
\mathbb{P}\left\{M_{\infty}>x\right\} \sim \bar{B}_{I}(x) /|b+c / \lambda| \quad \text { as } x \rightarrow \infty
$$

holds in the heavy-tailed case if and only if $B_{I}$ is subexponential.

The cases where $c>0$ or $Y_{1}$ takes values of both signs as well as the finite time horizon tail asymptotics for $M_{t}$ are more complicated-which is caused by dependence between the random variables $Y_{i}+c \tau_{i}$ and the process $N_{t}$ - and are described in the following theorem.

Theorem 1. Let $X_{t}$ be a compound renewal process with linear drift such that $a:=c+b \lambda<0$. Let the distribution $B$ of $Y_{1}^{+}$be strong subexponential and one of the following conditions hold:

(i) $c \leq 0$;

(ii) $c>0$ and $\mathbb{P}\{c \tau>x\}=o(\bar{B}(x))$ as $x \rightarrow \infty$. Then

$\mathbb{P}\left\{M_{t}>x\right\} \sim \frac{\lambda}{|a|} \int_{x}^{x+|a| \mathbb{E} N_{t} / \lambda} \bar{B}(v) d v \quad$ as $x \rightarrow \infty$ uniformly for all $t>0$.

In particular,

$$
\mathbb{P}\left\{M_{t}>x\right\} \sim \frac{\lambda}{|a|} \int_{x}^{x+|a| t} \bar{B}(v) d v \quad \text { as } x, t \rightarrow \infty .
$$

For a compound Poisson process with linear drift $X_{t}$ where $N_{t}$ is a homogeneous Poisson process with intensity of jumps $\lambda$, we have $\mathbb{E} N_{t}=t \lambda$ and $\mathbb{P}\{c \tau>x\}=e^{-\lambda x / c}=o(\bar{B}(x))$, so the following corollary. 
Corollary 2. Let $X_{t}$ be a compound Poisson process with linear drift. If $a=c+\lambda b<0$ and the distribution $B$ of $Y_{1}^{+}$is strong subexponential, then

$$
\mathbb{P}\left\{M_{t}>x\right\} \sim \frac{\lambda}{|a|} \int_{x}^{x+|a| t} \bar{B}(v) d v \quad \text { as } x \rightarrow \infty \text { uniformly for all } t>0 .
$$

A special case of Corollary 2 of $c<0$ and $Y_{1}>0$ was proven in Foss et al. (2013, Theorem 5.21) by alternative techniques in the context of the Cramér-Lundberg collective risk model. In the book by A. Borovkov and K. Borovkov (2008, Ch. 16) results for compound renewal processes with linear drift are only obtained for regularly varying distribution of $Y_{1}$; the corresponding techniques seems to be not applicable to general subexponential distributions.

If the linear drift coefficient $c$ is positive and if the condition $\mathbb{P}\{c \tau>x\}=$ $o(\bar{B}(x))$ of Theorem 1 fails, then the tail asymptotics of the distribution of $M_{t}$ may be more complicated. In particular, then $\mathbb{P}\left\{M_{t}>x\right\} \geq \mathbb{P}\left\{\tau_{1}>\right.$ $x / c\}$, so the tail of $M_{t}$ may be heavier than the integrated tail of $B$ if the tail of $\tau$ is so. We are not concerned with the tail asymptotics for $M_{t}$ in the general case when $c>0$; we only present the following result on the overall maximum $M_{\infty}$ which generalises (3) to an arbitrary value of $c$.

Theorem 3. Let $X_{t}$ be a compound renewal process such that $a:=c+b \lambda<0$ and the integrated tail distribution $F_{I}$ of $c \tau_{1}+Y_{1}^{+}$is subexponential. Then

$$
\mathbb{P}\left\{M_{\infty}>x\right\} \sim \frac{\lambda}{|a|} \bar{F}_{I}(x) \quad \text { as } x \rightarrow \infty .
$$

Proof. We only need to consider the case $c>0$. Then

$$
M_{\infty}=c \tau_{1}+\sup _{k \geq 0} \sum_{i=1}^{k}\left(Y_{i}+c \tau_{i+1}\right)
$$

and the result follows from (1) and from the observation that

$$
\mathbb{P}\left\{c \tau_{1}>x\right\}=O(\bar{F}(x))=o\left(\bar{F}_{I}(x)\right) \text { as } x \rightarrow \infty
$$

which allows to apply [14, Corollary 3.18]. The proof is complete.

Proof of Theorem 1. First let us prove that, for any fixed $t_{0}$, (4) holds uniformly for all $t \leq t_{0}$. Indeed, for all $t \leq t_{0}$,

$$
\mathbb{P}\left\{\sum_{n=1}^{N_{t}} Y_{n}>x+|c| t_{0}\right\} \leq \mathbb{P}\left\{M_{t}>x\right\} \leq \mathbb{P}\left\{\sum_{n=1}^{N_{t}} Y_{n}^{+}>x-|c| t_{0}\right\} .
$$


Since the $Y_{n}$ 's are strong subexponential, they are subexponential. For the renewal process $N_{t}$, there exists a $\delta>0$ such that $\mathbb{E}(1+\delta)^{N_{t_{0}}}<\infty$. Together with independence of the $Y_{n}$ 's and $N_{t}$, it allows us to apply Kesten's boundsee e.g. Foss et al. (2013, Theorem 3.34) - and to conclude the following uniform in $t \leq t_{0}$ analogue of the tail result for randomly stopped sums - see Foss et al. (2013, Theorem 3.37):

$$
\mathbb{P}\left\{\sum_{n=1}^{N_{t}} Y_{n}>x\right\} \sim \mathbb{E} N_{t} \mathbb{P}\left\{Y_{1}>x\right\} \quad \text { as } x \rightarrow \infty \text { uniformly for all } t \leq t_{0} .
$$

The same arguments work for the $Y_{n}^{+}$'s. Then, taking into account that subexponentiality of $Y_{n}$ 's implies $B$ is long-tailed, we conclude from (5) that

$$
\mathbb{P}\left\{M_{t}>x\right\} \sim \mathbb{E} N_{t} \mathbb{P}\left\{Y_{1}>x\right\} \quad \text { as } x \rightarrow \infty \text { uniformly for all } t \leq t_{0},
$$

which is equivalent to the fact that (4) holds uniformly for all $t \leq t_{0}$ because

$$
\frac{\lambda}{|a|} \int_{x}^{x+|a| \mathbb{E} N_{t} / \lambda} \bar{B}(v) d v \sim \mathbb{E} N_{t} \bar{B}(x) \text { as } x \rightarrow \infty \text { uniformly for all } t \leq t_{0},
$$

again by long-tailedness of $B$.

Therefore there exists an increasing function $h(x) \rightarrow \infty$ such that (4) holds uniformly for all $t \leq h(x)$. Then it remains to prove (4) for the range $t>h(x)$ where the above arguments clearly do not help. Instead, we proceed with a natural technique of getting the lower and upper bounds for the tail of $M_{t}$ which are asymptotically equivalent. For the lower bound, fix an $\varepsilon>0$. By the strong law of large numbers, there exists an $A$ such that

$$
\mathbb{P}\left\{\left|T_{n}-n \mathbb{E} \tau\right|<n \varepsilon+A \text { for all } n \geq 1\right\} \geq 1-\varepsilon .
$$

Notice that

$$
\mathbb{P}\left\{M_{t}>x\right\} \geq \mathbb{P}\left\{\sum_{i=1}^{n} Y_{i}+c T_{n}>x \text { for some } n \leq N_{t}\right\} .
$$

On the event (6), if $t \geq n(\mathbb{E} \tau+\varepsilon)+A$ (equivalently, $n \leq n(t):=\left[\frac{t-A}{\mathbb{E} \tau+\varepsilon}\right]$ ) then $T_{n} \leq t$ and hence $N_{t} \geq n$. Then independence of the jumps $Y_{n}$ 's and the renewal process $N_{s}$ yields the following lower bound

$\mathbb{P}\left\{M_{t}>x\right\} \geq(1-\varepsilon) \mathbb{P}\left\{\sum_{i=1}^{n} Y_{i}+c(n(\mathbb{E} \tau+\varepsilon)+A)>x\right.$ for some $\left.n \leq n(t)\right\}$ 
for $c \leq 0$ and

$$
\mathbb{P}\left\{M_{t}>x\right\} \geq(1-\varepsilon) \mathbb{P}\left\{\sum_{i=1}^{n} Y_{i}+c(n(\mathbb{E} \tau-\varepsilon)-A)>x \text { for some } n \leq n(t)\right\}
$$

for $c>0$. Thus, in both cases,

$$
\mathbb{P}\left\{M_{t}>x\right\} \geq(1-\varepsilon) \mathbb{P}\left\{\max _{n \leq n(t)} \sum_{i=1}^{n}\left(Y_{i}+c \mathbb{E} \tau-|c| \varepsilon\right)>x+|c| A\right\} .
$$

Applying the equivalence (2) we obtain the following lower bound:

$$
\begin{aligned}
& \mathbb{P}\left\{M_{t}>x\right\} \geq \frac{1-\varepsilon+o(1)}{|b+c \mathbb{E} \tau-| c|\varepsilon|} \int_{x+|c| A}^{x+|c| A+n(t)|b+c \mathbb{E} \tau-| c|\varepsilon|} \bar{B}(v) d v \\
& \sim \frac{1-\varepsilon}{|a / \lambda-| c|\varepsilon|} \int_{0}^{t \frac{|a / \lambda-| c|\varepsilon|}{\mathbb{E} \tau+\varepsilon}} \bar{B}(x+v) d v \quad \text { as } x, t \rightarrow \infty,
\end{aligned}
$$

because $B$ is a long-tailed distribution. Taking into account that, for all $\gamma>0$

$$
\int_{0}^{\gamma t} \bar{B}(x+u) d u \geq \min (1, \gamma) \int_{0}^{t} \bar{B}(x+u) d u
$$

and letting $\varepsilon \downarrow 0$ we conclude the lower bound

$$
\mathbb{P}\left\{M_{t}>x\right\} \geq \frac{\lambda+o(1)}{|a|} \int_{x}^{x+|a| t} \bar{B}(v) d v \quad \text { as } x, t \rightarrow \infty .
$$

Now let us turn to the upper bound for $\mathbb{P}\left\{M_{t}>x\right\}$. First consider the case $c \leq 0$ when the trajectory of $X_{t}$ linearly drops down between jumps and the maximum may be only attained at a jump epoch,

$$
M_{t}=\max _{0 \leq n \leq N_{t}} \sum_{i=1}^{n}\left(Y_{i}+c \tau_{i}\right)
$$

Therefore, for any $\varepsilon>0$,

$$
\begin{aligned}
\mathbb{P}\left\{M_{t}>x\right\} \leq \mathbb{P}\left\{\max _{n \leq(1+\varepsilon) \mathbb{E} N_{t}} \sum_{i=1}^{n}\left(Y_{i}+c \tau_{i}\right)>x\right\} \\
\quad+\mathbb{P}\left\{\sum_{i=1}^{N_{t}} Y_{i}^{+}>x, N_{t}>(1+\varepsilon) \mathbb{E} N_{t}\right\} .
\end{aligned}
$$


The distribution of $Y$ is strong subexponential and $c<0$, so $Y+c \tau$ is strong subexponential too and $\mathbb{P}\{Y+c \tau>x\} \sim \mathbb{P}\{Y>x\}=\bar{B}(x)$ as $x \rightarrow \infty$. Thus, by (2),

$$
\begin{aligned}
\mathbb{P}\left\{\max _{n \leq(1+\varepsilon) \mathbb{E} N_{t}} \sum_{i=1}^{n}\left(Y_{i}+c \tau_{i}\right)>x\right\} & \sim \frac{\lambda}{|a|} \int_{x}^{x+|a|(1+\varepsilon) \mathbb{E} N_{t} / \lambda} \bar{B}(y) d y \\
& \leq \frac{\lambda(1+\varepsilon)}{|a|} \int_{x}^{x+|a| \mathbb{E} N_{t} / \lambda} \bar{B}(y) d y
\end{aligned}
$$

because $\bar{B}(y)$ is decreasing. Further,

$$
\begin{aligned}
\mathbb{P}\left\{\sum_{i=1}^{N_{t}} Y_{i}^{+}>x,\right. & \left.N_{t}>(1+\varepsilon) \mathbb{E} N_{t}\right\} \\
= & \sum_{k=1}^{\infty} \mathbb{P}\left\{\sum_{i=1}^{N_{t}} Y_{i}^{+}>x,(1+k \varepsilon) \mathbb{E} N_{t}<N_{t} \leq(1+(k+1) \varepsilon) \mathbb{E} N_{t}\right\} \\
& \leq \sum_{k=1}^{\infty} \mathbb{P}\left\{\sum_{i=1}^{(1+(k+1) \varepsilon) \mathbb{E} N_{t}} Y_{i}^{+}>x\right\} \mathbb{P}\left\{N_{t}>(1+k \varepsilon) \mathbb{E} N_{t}\right\},
\end{aligned}
$$

owing to independence of $Y_{n}$ 's and $N_{t}$. Denote $K:=\left[(1+k \varepsilon) \mathbb{E} N_{t}\right]$. Then, for $\varepsilon \in(0,1)$,

$$
\begin{aligned}
\mathbb{P}\left\{N_{t}>(1+k \varepsilon) \mathbb{E} N_{t}\right\} & =\mathbb{P}\left\{T_{K} \leq t\right\} \\
& \leq \mathbb{P}\left\{K \mathbb{E} \tau(1-\varepsilon / 2)-T_{K} \geq 0\right\}
\end{aligned}
$$

for sufficiently large $t$ because, as $t \rightarrow \infty$,

$$
K \mathbb{E} \tau(1-\varepsilon / 2)-t \sim t((1+k \varepsilon)(1-\varepsilon / 2)-1) \geq t\left(\varepsilon / 2-\varepsilon^{2} / 2\right)>0 .
$$

Since the random variable $\mathbb{E} \tau(1-\varepsilon / 2)-\tau$ has negative expectation $-\varepsilon \mathbb{E} \tau / 2$ and is bounded above by $\mathbb{E} \tau(1-\varepsilon / 2)$, there exists a $\beta=\beta(\varepsilon)>0$ such that

$$
\mathbb{E} e^{\beta(\mathbb{E} \tau(1-\varepsilon / 2)-\tau)}=1-\delta<1 .
$$

Hence, by exponential Chebyshev's inequality,

$$
\mathbb{P}\left\{K \mathbb{E} \tau(1-\varepsilon / 2)-T_{K} \geq 0\right\} \leq(1-\delta)^{K}
$$

for all $k \geq 1$ and sufficiently large $t$, so

$$
\mathbb{P}\left\{N_{t}>(1+k \varepsilon) \mathbb{E} N_{t}\right\} \leq(1-\delta)^{\left[(1+k \varepsilon) \mathbb{E} N_{t}\right]} .
$$


By Kesten's bound there is an $A<\infty$ such that

$$
\mathbb{P}\left\{\sum_{i=1}^{(1+(k+1) \varepsilon) \mathbb{E} N_{t}} Y_{i}^{+}>x\right\} \leq A(1+\delta / 8)^{(1+(k+1) \varepsilon) \mathbb{E} N_{t}} \mathbb{P}\{Y>x\}
$$

for all $x>0, k \geq 1$ and $t>0$. For $k \geq 1$ and sufficiently large $t$,

$$
(1+(k+1) \varepsilon) \mathbb{E} N_{t} \leq 2\left[(1+k \varepsilon) \mathbb{E} N_{t}\right]
$$

thus

$$
\begin{aligned}
\mathbb{P}\left\{\sum_{i=1}^{(1+(k+1) \varepsilon) \mathbb{E} N_{t}} Y_{i}^{+}>x\right\} & \leq A(1+\delta / 8)^{2\left[(1+k \varepsilon) \mathbb{E} N_{t}\right]} \mathbb{P}\{Y>x\} \\
& \leq A(1+\delta / 2)^{\left[(1+k \varepsilon) \mathbb{E} N_{t}\right]} \mathbb{P}\{Y>x\}
\end{aligned}
$$

Substituting (10) and (11) into (9) and taking into account that $(1-\delta)(1+$ $\delta / 2) \leq 1-\delta / 2$, we obtain, for all sufficiently large $t$,

$\mathbb{P}\left\{\sum_{i=1}^{N_{t}} Y_{i}^{+}>x, N_{t}>(1+\varepsilon) \mathbb{E} N_{t}\right\} \leq A \mathbb{P}\{Y>x\} \sum_{k=1}^{\infty}(1-\delta / 2)^{\left[(1+k \varepsilon) \mathbb{E} N_{t}\right]}$.

The sum on the right goes to zero as $t \rightarrow \infty$. Therefore, for any fixed $\varepsilon>0$,

$$
\mathbb{P}\left\{\sum_{i=1}^{N_{t}} Y_{i}^{+}>x, N_{t}>(1+\varepsilon) \mathbb{E} N_{t}\right\}=o(\mathbb{P}\{Y>x\}) \quad \text { as } t \rightarrow \infty
$$

uniformly for all $x>0$. Combining this bound with (8) we get

$$
\mathbb{P}\left\{M_{t}>x\right\} \leq \frac{\lambda(1+\varepsilon)+o(1)}{|a|} \int_{x}^{x+|a| \mathbb{E} N_{t} / \lambda} \bar{B}(y) d y \quad \text { as } x \rightarrow \infty
$$

uniformly for all $t \geq h(x)$. Letting $\varepsilon \downarrow 0$, we conclude the desired upper bound which proves Theorem 1 in the case $c \leq 0$.

Now consider the case $c>0$ when the trajectory of $X_{t}$ linearly grows between jump epochs $T_{n}$ and the maximum may be only attained just prior to a jump epoch or at time $t$, hence

$$
M_{t} \leq c \tau_{1}+\max _{0 \leq n \leq N_{t}} \sum_{i=1}^{n}\left(Y_{i}+c \tau_{i+1}\right)=: c \tau_{1}+\widehat{M}_{t}
$$


where $\tau_{1}$ and $\widehat{M}_{t}$ are independent. The distribution of $Y$ is strong subexponential and the tail of $c \tau$ is of order $o(\bar{B}(x))$, so $Y+c \tau$ is strong subexponential too and $\mathbb{P}\{Y+c \tau>x\} \sim \mathbb{P}\{Y>x\}=\bar{B}(x)$ as $x \rightarrow \infty$. Thus, similar to the case $c \leq 0$, we get that

$$
\mathbb{P}\left\{\widehat{M}_{t}>x\right\} \leq \frac{\lambda+o(1)}{|a|} \int_{x}^{x+|a| \mathbb{E} N_{t} / \lambda} \bar{B}(y) d y \quad \text { as } x \rightarrow \infty
$$

uniformly for all $t \geq h(x)$. Since $c \tau_{1}$ and $\widehat{M}_{t}$ in (12) are independent,

$$
\mathbb{P}\left\{M_{t}>x\right\} \leq \mathbb{P}\left\{c \tau_{1}>x\right\}+\int_{0}^{x} \mathbb{P}\left\{c \tau_{1} \in d u\right\} \mathbb{P}\left\{\widehat{M}_{t}>x-u\right\}
$$

which allows to carry out standard calculations for subexponential distributions based on the condition $\mathbb{P}\left\{c \tau_{1}>x\right\}=o(\bar{B}(x))$ and the upper bound for $\widehat{M}_{t}$ and to conclude the upper bound

$$
\mathbb{P}\left\{M_{t}>x\right\} \leq \frac{\lambda+o(1)}{|a|} \int_{x}^{x+|a| \mathbb{E} N_{t} / \lambda} \bar{B}(y) d y \quad \text { as } x \rightarrow \infty,
$$

which completes the proof in the case $c>0$. The proof of Theorem 1 is complete.

We conclude this section with the following theorem which is nothing other than the principle of a single big jump for the maximum $M_{t}$. For $A>0$ and $\varepsilon>0$ consider events

$$
D_{k}:=\left\{\left|X_{s}-a s\right| \leq \varepsilon s+A \text { for all } s<T_{k}, Y_{k}>x+|a| T_{k}\right\}
$$

which, for large $x$, roughly speaking means that up to time $T_{k}$ the process $X_{s}$ drifts down with rate $a$ according to the strong law of large numbers and then makes a big jump up at time $T_{k}$ of size $x$ plus value that compensates the negative drift up to this time. As stated in the next theorem, the union of these events describes the most probable way by which large deviations of $M_{t}$ can occur - it is very different from what would be observed if the distribution of $Y_{n}$ was light-tailed. It is an analogue for discrete time process of the principle of a single big jump for the maximum of a random walk with negative drift, see Theorem 5.4 in Foss et al. (2013).

Theorem 4. Under the conditions of Theorem 1, for any fixed $\varepsilon>0$,

$$
\lim _{A \rightarrow \infty} \lim _{t, x \rightarrow \infty} \mathbb{P}\left\{\cup_{k=1}^{N_{t}} D_{k} \mid M_{t}>x\right\} \geq \frac{|a|}{|a|+2 \varepsilon} .
$$


Proof. Take $\gamma=1 / \lambda(1+|a|)$. For $k$ satisfying $k(\mathbb{E} \tau+\gamma \varepsilon)+A \leq t$, each of the events

$$
\begin{gathered}
\widetilde{D}_{k}:=\left\{\left|X_{s}-a s\right| \leq \varepsilon s+A \text { for all } s<T_{k}, T_{j} \leq j(\mathbb{E} \tau+\varepsilon \gamma)+A \text { for all } j \geq 1,\right. \\
\left.M_{T_{k}-0} \leq x, Y_{k}>x+A+T_{k}(|a|+\varepsilon)\right\}
\end{gathered}
$$

is contained in $\left\{T_{k} \leq t\right\}$ and in $D_{k}$ and implies that $M_{T_{k}}>x$ because on the event $\widetilde{D}_{k}$ we have

$$
X_{T_{k}}=X_{T_{k}-0}+Y_{k}>(a-\varepsilon) T_{k}-A+x+A+T_{k}(|a|+\varepsilon)=x,
$$

so that $M_{t}>x$. Then, for $N=N(t):=\left[\frac{t-A}{\mathbb{E} \tau+\gamma \varepsilon}\right]$, we have that $N_{t} \geq N$ on all $\widetilde{D}_{k}$, hence

$$
\begin{aligned}
\mathbb{P}\left\{\cup_{k=1}^{N_{t}} D_{k} \mid M_{t}>x\right\} & \geq \mathbb{P}\left\{\cup_{k=1}^{N} \widetilde{D}_{k}, N_{t} \geq N \mid M_{t}>x\right\} \\
& =\frac{\mathbb{P}\left\{\cup_{k=1}^{N} \widetilde{D}_{k}\right\}}{\mathbb{P}\left\{M_{t}>x\right\}} .
\end{aligned}
$$

The events $\widetilde{D}_{k}$ are disjoint, hence

$$
\mathbb{P}\left\{\cup_{k=1}^{N} \widetilde{D}_{k}\right\}=\sum_{k=1}^{N} \mathbb{P}\left\{\widetilde{D}_{k}\right\}
$$

It follows from the strong law of large numbers applied to both $X_{s}$ and $N_{s}$ that, for any fixed $\delta>0$, there exists an $A$ such that, for all $x>A$,

$$
\begin{aligned}
& \mathbb{P}\left\{\cup_{k=1}^{N} \widetilde{D}_{k}\right\} \\
& \geq(1-\delta / 4) \sum_{k=1}^{N} \mathbb{P}\left\{Y_{k}>x+A+T_{k}(|a|+\varepsilon) \mid T_{j} \leq j(\mathbb{E} \tau+\varepsilon \gamma)+A \text { for all } j \geq 1\right\} \\
& \quad \geq(1-\delta / 4) \sum_{k=1}^{N} \mathbb{P}\left\{Y_{k}>x+(1+|a|+\varepsilon) A+k(|a|+\varepsilon)(\mathbb{E} \tau+\varepsilon \gamma)\right\} \\
& \quad \geq(1-\delta / 4) \sum_{k=1}^{N} \mathbb{P}\left\{Y_{k}>x+(1+|a|+\varepsilon) A+k(|a|+2 \varepsilon) / \lambda\right\},
\end{aligned}
$$

by the choice of the $\gamma>0$. Since the distribution $B$ is long-tailed,

$$
\mathbb{P}\left\{\cup_{k=1}^{N} \widetilde{D}_{k}\right\} \quad \geq(1-\delta / 2) \sum_{k=0}^{N-1} \mathbb{P}\left\{Y_{k}>x+k(|a|+2 \varepsilon) / \lambda\right\}
$$


for all sufficiently large $x$. Hence

$$
\mathbb{P}\left\{\cup_{k=0}^{N-1} \widetilde{D}_{k}\right\} \geq \frac{1-\delta / 2}{(|a|+2 \varepsilon) / \lambda} \int_{x}^{x+N(|a|+2 \varepsilon) / \lambda} \bar{B}(y) d y,
$$

because $\bar{B}(y)$ decreases. Take also into account that, as $t \rightarrow \infty$,

$$
\frac{N(|a|+2 \varepsilon)}{\lambda}=\frac{N(t)(|a|+2 \varepsilon)}{\lambda} \sim t \frac{|a|+2 \varepsilon}{1+\lambda \gamma \varepsilon} \geq t(|a|+\varepsilon),
$$

owing the choice of the $\gamma>0$, so we deduce

$$
\mathbb{P}\left\{\cup_{k=1}^{N} \widetilde{D}_{k}\right\} \geq \frac{1-\delta / 2}{(|a|+2 \varepsilon) / \lambda} \int_{x}^{x+t|a|} \bar{B}(y) d y .
$$

Substituting this estimate and the asymptotics for $M_{t}$ into (14) we conclude

$$
\lim _{t, x \rightarrow \infty} \mathbb{P}\left\{\cup_{k=1}^{N_{t}} D_{k} \mid M_{t}>x\right\} \geq \frac{(1-\delta)|a|}{|a|+2 \varepsilon} .
$$

Now we can make $\delta>0$ as small as we please by choosing a sufficiently large $A$. This completes the proof.

\section{Asymptotics for Lévy processes}

Let $X_{t}$ be a càdlàg stochastic process in $\mathbb{R}$ which means that its paths are right continuous with left limits everywhere, with probability 1 . Then the supremum

$$
M_{t}:=\sup _{u \in[0, t]} X_{u}
$$

is finite a.s. for all $t$. In this section we study tail behaviour of the distribution of $M_{t}$ for a Lévy process $X_{t}$ starting at the origin. Our main result here is the following theorem.

Theorem 5. Assume the finite mean and negative drift, $a:=\mathbb{E} X_{1}<0$. If the integrated tail distribution $F_{I}$ of $X_{1}$ is subexponential, then

$$
\mathbb{P}\left\{\max _{u>0} X_{u}>x\right\} \sim \frac{1}{|a|} \int_{x}^{\infty} \bar{F}(v) d v \quad \text { as } x \rightarrow \infty .
$$

If the distribution $F$ of $X_{1}$ is strong subexponential, then, uniformly for all $t>0$,

$$
\mathbb{P}\left\{\max _{u \in[0, t]} X_{u}>x\right\} \sim \frac{1}{|a|} \int_{x}^{x+t|a|} \bar{F}(v) d v \quad \text { as } x \rightarrow \infty
$$


It has been suggested by Asmussen and Klüppelberg (1996, Sect. 1.1) and by Asmussen (1998, Sect. 2.4) to follow a discrete skeleton argument in order to prove this asymptotics for $t=\infty$ when the integrated tail of the Lévy measure is subexponential; notice that this approach requires additional considerations which take into account fluctuations of Lévy processes within time slots; see the remark after Theorem 6 .

In Braverman et al. (2002) tail asymptotics are presented for some subclass of subadditive functionals of Lévy process with regularly varying at infinity Lévy measure. The overall supremum is a particular example considered in that article. Clearly the problem for (sub)additive functionals becomes much more difficult beyond the case of regularly varying distributions. Neither technique developed for regularly varying case nor in this paper is then applicable. There is no a suitable technique even for additive functionals of random walks. For instance, the tail asymptotics for the maximum of the area under the graph of a random walk (a Lévy process) is quite different to that for regularly varying distributions if the jump tail distribution is of Weibullian type with a shape parameter greater than $1 / 2$.

In Klüppelberg et al. (2004, Theorem 6.2), tail asymptotics for the overall supremum of negatively driven Lévy process are derived via direct approach based on ladder properties of the Lévy process.

In Doney et al. (2016) the passage time problem is considered for Lévy processes, emphasising heavy tailed cases; local and functional versions of limit distributions are derived for the passage time itself, as well as for the position of the process just prior to passage, and the overshoot of a high level which is an extension for Lévy processes of corresponding results for random walks, see e.g. Foss et al. (2013, Theorem 5.24).

In Foss et al. (2007, Theorem 3.1), a Markov modulated Lévy process is studied and again the tail asymptotics for the overall supremum were proven, via reduction to a Markov modulated random walk.

In the book by A. Borovkov and K. Borovkov (2008, Ch. 15) some partial results on $\max _{u \in[0, t]} X_{u}$ are formulated (see, for example, Theorems 15.2.2(vi) and 15.3.12 there) under some specific conditions on the distribution of $X_{1}$; the supporting arguments provided may be hardly considered as clear and comprehensive. For example, on page 525 the authors justify transition from integer $t$ to non-integer $t$ by convergence in probability $X_{u} \rightarrow 0$ as $u \rightarrow \infty$ which is clearly insufficient. Also notice that it was not proven there that the corresponding asymptotics hold uniformly for all $t>0$.

Related results on sample-path large deviations of scaled Lévy processes $X(n t) / n$ with regularly varying Lévy measure are proven by Rhee et al. (2016). 
The following result is due to Willekens [24]; it was proven via natural elementary rather short arguments.

Theorem 6. Let $X_{t}$ be a Lévy process. For any fixed $t>0$, the following assertions are equivalent:

(i) the distribution of $X_{t}$ is long-tailed;

(ii) the distribution of $M_{t}$ is long-tailed.

Each of (i) and (ii) implies

$$
\mathbb{P}\left\{M_{t}>x\right\} \sim \mathbb{P}\left\{X_{t}>x\right\} \quad \text { as } x \rightarrow \infty .
$$

Notice that Theorem 6 together with Theorem 1 for regenerative processes from Palmowski and Zwart (2007) — or with Theorem 3.3 from Asmussen et al. (1999) - provides a correct version of skeleton approach for proving subexponential asymptotics for the overall supremum $M_{\infty}$ under negative drift assumption.

To prove Theorem 5 we need the following lemma which may be of independent interest. A similar proposition was proven for subexponential distributions in Embrechts et al. (1979, Proposition 1); the proof provided there does not work for long-tailed distributions.

Lemma 7. Let $G$ and $B$ be two distributions on $\mathbb{R}$ and let $G$ be light-tailed, that is, there exist $\lambda>0$ and $c<\infty$ such that $\bar{G}(x) \leq c e^{-\lambda x}$ for all $x$. Denote $F:=G * B$.

(i) If $B$ is long-tailed then $\bar{F}(x) \sim \bar{B}(x)$ as $x \rightarrow \infty$; in particular, $F$ is long-tailed too.

(ii) If $F$ is long-tailed then $B$ is long-tailed too.

Proof. (i) Assume that $B$ is long-tailed. Then there exists an $x_{0}$ such that $\bar{B}(x-1) \leq \bar{B}(x) e^{\lambda / 2}$ for all $x \geq x_{0}$. Therefore, there exists a $c<\infty$ such that

$$
\bar{B}(x-y) \leq \bar{B}(x) \max \left\{1, c e^{\lambda y / 2}\right\} \quad \text { for all } x, y \in \mathbb{R}
$$

hence

$$
\frac{\bar{F}(x)}{\bar{B}(x)}=\int_{-\infty}^{\infty} \frac{\bar{B}(x-y)}{\bar{B}(x)} G(d y) \rightarrow 1 \quad \text { as } x \rightarrow \infty
$$

by the dominated convergence theorem because, for all $y, \bar{B}(x-y) / \bar{B}(x) \rightarrow$ 1 as $x \rightarrow \infty$ and the function $\max \left\{1, c e^{\lambda y / 2}\right\}$ is $G$-integrable due to the condition $\bar{G}(x)=O\left(e^{-\lambda x}\right)$. 
(ii) Assume that $F$ is long-tailed. Let us then prove that $\bar{B}(x) \sim \bar{F}(x)$ which implies long-tailedness of $B$. For all $x$ and $h \in \mathbb{R}$, the following inequality holds:

$$
\bar{F}(x-h)=\overline{G * B}(x-h) \geq \bar{G}(-h) \bar{B}(x) .
$$

Therefore, long-tailedness on $F$ implies

$$
\lim _{x \rightarrow \infty} \bar{B}(x) / \bar{F}(x)=\lim _{x \rightarrow \infty} \bar{B}(x) / \bar{F}(x-h) \leq 1 / \bar{G}(-h),
$$

so $\bar{B}(x) \leq(1+o(1)) \bar{F}(x)$ as $x \rightarrow \infty$. Then it remains to prove that

$$
\liminf _{x \rightarrow \infty} \bar{B}(x) / \bar{F}(x) \geq 1 .
$$

Suppose it does not hold. Then there exist an $\varepsilon>0$ and a sequence $x_{n} \rightarrow \infty$ such that

$$
\bar{B}\left(x_{n}\right) \leq(1-\varepsilon) \bar{F}\left(x_{n}\right) \text { for all } n \geq 1
$$

We have

$$
\begin{aligned}
\bar{F}\left(x_{n}+h\right) & =\int_{-\infty}^{h} \bar{B}\left(x_{n}+h-y\right) G(d y)+\int_{h}^{\infty} \bar{B}\left(x_{n}+h-y\right) G(d y) \\
& \leq \bar{B}\left(x_{n}\right)+\int_{h}^{\infty} \bar{B}\left(x_{n}+h-y\right) G(d y) \\
& \leq(1-\varepsilon) \bar{F}\left(x_{n}\right)+\frac{1}{\bar{G}(-h)} \int_{h}^{\infty} \bar{F}\left(x_{n}-y\right) G(d y),
\end{aligned}
$$

by (18) and (16). Since $F$ is long-tailed, by part (i), the integral on the right hand side is asymptotically equivalent to $\bar{F}\left(x_{n}\right) \bar{G}(h)$ as $n \rightarrow \infty$, hence

$$
1=\lim _{n \rightarrow \infty} \bar{F}\left(x_{n}+h\right) / \bar{F}\left(x_{n}\right) \leq 1-\varepsilon+\bar{G}(h) / \bar{G}(-h) .
$$

Letting $h \rightarrow \infty$ leads to the contradiction $1 \leq 1-\varepsilon$ and (17) is proven.

Given the distribution of $X_{1}$ is infinitely divisible, recall the LévyKhintchine formula for its characteristic exponent $\Psi(\theta):=\log \mathbb{E} e^{i \theta X_{1}}$, for all $\theta \in \mathbb{R}$,

$$
\begin{aligned}
& \Psi(\theta) \\
& \quad=\quad\left(i \alpha \theta-\frac{1}{2} \sigma^{2} \theta^{2}\right)+\int_{0<|x|<1}\left(e^{i \theta x}-1-i \theta x\right) \Pi(d x)+\int_{|x| \geq 1}\left(e^{i \theta x}-1\right) \Pi(d x) \\
& =: \quad \Psi_{1}(\theta)+\Psi_{2}(\theta)+\Psi_{3}(\theta) ;
\end{aligned}
$$


see, e.g. Kyprianou (2006, Sect. 2.1). Here $\Pi$ is the Lévy measure concentrated on $\mathbb{R} \backslash\{0\}$ and satisfying $\int_{\mathbb{R}}\left(1 \wedge x^{2}\right) \Pi(d x)<\infty$. Let $X_{t}^{(1)}, X_{t}^{(2)}$ and $X_{t}^{(3)}$ be independent processes given in the Lévy-Itô decomposition $X_{t} \stackrel{d}{=} X_{t}^{(1)}+X_{t}^{(2)}+X_{t}^{(3)}$, where $X_{t}^{(1)}$ is a linear Brownian motion with characteristic exponent given by $\Psi^{(1)}, X_{t}^{(2)}$ is a square integrable martingale with an almost surely countable number of jumps on each finite time interval which are of magnitude less than unity and with characteristic exponent given by $\Psi^{(2)}$ and $X_{t}^{(3)}$ is a compound Poisson process with intensity $\Pi(\mathbb{R} \backslash(-1,1))$ and jump distribution $\frac{\Pi(d x)}{\Pi(\mathbb{R} \backslash(-1,1))}$ concentrated on $\mathbb{R} \backslash(-1,1)$. It is known - see, e.g. Kyprianou (2006, Theorem 3.6) or Sato (1999, Theorem 25.17) — that $Z_{t}:=X_{t}^{(1)}+X_{t}^{(2)}$ possesses all exponential moments finite which allows to prove the following corollary from Lemma 7.

Corollary 8. (i) The distribution of $X_{1}$ is long-tailed if and only if the distribution of $X_{1}^{(3)}$ is so. In both cases, $\mathbb{P}\left\{X_{1}>x\right\} \sim \mathbb{P}\left\{X_{1}^{(3)}>x\right\}$ as $x \rightarrow \infty$.

(ii) The distribution of $X_{1}^{+}$is strong subexponential if and only if the distribution $\Pi(d x) / \Pi(1, \infty)$ concentrated on $(1, \infty)$ is so. In both cases, $\mathbb{P}\left\{X_{1}>x\right\} \sim \Pi(x, \infty)$ as $x \rightarrow \infty$.

Proof. The assertion (i) is immediate from Lemma 7.

(ii) If the distribution of $X_{1}^{+}$is strong subexponential, then it is longtailed, so that $\mathbb{P}\left\{X_{1}>x\right\} \sim \mathbb{P}\left\{X_{1}^{(3)}>x\right\}$ as $x \rightarrow \infty$. Hence, the distribution of $X_{1}^{(3)+}$ is strong subexponential too. Since the distribution of $X_{1}^{(3)+}$ is compound Poisson with parameter $\Pi(1, \infty)$ and jump distribution $\Pi(d x) / \Pi(1, \infty)$ concentrated on $(1, \infty)$, Theorem 3 of Foss et al. (2013) yields that $\mathbb{P}\left\{X_{1}>x\right\} \sim \Pi(x, \infty)$. Therefore, the distribution $\Pi(d x) / \Pi(1, \infty)$ is strong subexponential - see, e.g. Foss et al. (2013, Corollary 3.26$)$.

If the distribution $\Pi(d x) / \Pi(1, \infty)$ concentrated on $(1, \infty)$ is strong subexponential, then $\mathbb{P}\left\{X_{1}>x\right\} \sim \mathbb{P}\left\{X_{1}^{(3)+}>x\right\} \sim \Pi(x, \infty)$ by the theorem on tail behavior for random sums - see e.g. Foss et al. (2013, Theorem 3.37 ).

In the sequel we need an accurate upper bound for $\mathbb{E} e^{s X_{t}^{(2)}}$. By the condition $c:=\int_{(-1,1)} x^{2} \Pi(d x)<\infty$ we may produce the following upper 
bound:

$$
\begin{aligned}
\int_{(-1,1)}\left(e^{s x}-1-s x\right) \Pi(d x) & =\int_{(-1,1)} \sum_{k=2}^{\infty} \frac{(s x)^{k}}{k !} \Pi(d x) \\
& \leq \sum_{k=2}^{\infty} \frac{s^{k}}{k !} \int_{(-1,1)} x^{2} \Pi(d x)=c\left(e^{s}-1-s\right) .
\end{aligned}
$$

Therefore,

$$
\mathbb{E} e^{s X_{t}^{(2)}}=e^{t \int_{(-1,1)}\left(e^{s x}-1-s x\right) \Pi(d x)} \leq e^{c t e^{s}} .
$$

Proof of Theorem 5. We start with a lower bound. We have $a=\mathbb{E} X_{1}^{(3)}+$ $\mathbb{E} Z_{1}$. Fix $\varepsilon>0$ and consider two independent processes

$$
X_{t}^{\varepsilon}:=X_{t}^{(3)}+t \mathbb{E} Z_{1}-t \varepsilon \quad \text { and } \quad Z_{t}^{\varepsilon}:=Z_{t}-t \mathbb{E} Z_{1}+t \varepsilon,
$$

so that $X_{t}=X_{t}^{\varepsilon}+Z_{t}^{\varepsilon}$. Then, for all $x$ and $y>0$,

$$
\mathbb{P}\left\{\max _{u \in[0, t]} X_{u}>x\right\} \geq \mathbb{P}\left\{\max _{u \in[0, t]} X_{u}^{\varepsilon}>x+y\right\} \mathbb{P}\left\{\inf _{u \geq 0} Z_{u}^{\varepsilon}>-y\right\} .
$$

The drift of the process $Z_{t}^{\varepsilon}$ is $t \varepsilon>0$. Thus the overall minimum of the process $Z_{t}^{\varepsilon}$ is finite with probability 1 , so there exists an $y_{0}>0$ such that

$$
\mathbb{P}\left\{\inf _{u \geq 0} Z_{u}^{\varepsilon}>-y_{0}\right\} \geq 1-\varepsilon,
$$

which implies, for all $t>0$,

$$
\mathbb{P}\left\{\max _{u \in[0, t]} X_{u}>x\right\} \geq(1-\varepsilon) \mathbb{P}\left\{\max _{u \in[0, t]} X_{u}^{\varepsilon}>x+y_{0}\right\} .
$$

Since $X_{1}^{+}$is assumed to be strong subexponential, by Corollary 8 the distribution $\Pi(d x) / \Pi(1, \infty)$ on $(1, \infty)$ is strong subexponential too and $\bar{\Pi}(x) \sim$ $\mathbb{P}\left\{X_{1}>x\right\}=\bar{F}(x)$ as $x \rightarrow \infty$. Then the compound Poisson process $X_{t}^{\varepsilon}$ with intensity $\Pi(\mathbb{R} \backslash(-1,1))$ and drift $(a-\varepsilon) t$ satisfies all the conditions of Corollary 2 which implies, as $x \rightarrow \infty$ uniformly for all $t>0$,

$$
\mathbb{P}\left\{\max _{u \in[0, t]} X_{u}^{\varepsilon}>x\right\} \sim \frac{1}{|a-\varepsilon|} \int_{x}^{x+t|a-\varepsilon|} \bar{F}(v) d v \geq \frac{1}{|a-\varepsilon|} \int_{x}^{x+t|a|} \bar{F}(v) d v
$$

Letting $\varepsilon \downarrow 0$, we conclude from (20) the lower bound

$$
\mathbb{P}\left\{\max _{u \in[0, t]} X_{u}>x\right\} \geq \frac{1+o(1)}{|a|} \int_{x}^{x+t|a|} \bar{F}(v) d v \quad \text { as } x \rightarrow \infty .
$$


Now let us prove an upper bound. Consider two independent processes

$$
X_{t}^{\varepsilon}:=X_{t}^{(3)}+t \mathbb{E} Z_{1}+t \varepsilon \quad \text { and } \quad Z_{t}^{\varepsilon}:=Z_{t}-t \mathbb{E} Z_{1}-t \varepsilon,
$$

so that $X_{t}=X_{t}^{\varepsilon}+Z_{t}^{\varepsilon}$. Then

$$
\max _{u \in[0, t]} X_{u} \leq \max _{u \in[0, t]} X_{u}^{\varepsilon}+\max _{u \in[0, t]} Z_{u}^{\varepsilon}
$$

The drift of $Z_{t}^{\varepsilon}$ is negative, $\mathbb{E} Z_{t}^{\varepsilon}=-t \varepsilon$, so the overall supremum of $Z_{t}^{\varepsilon}$ is finite a.s. Since all positive exponential moments of $Z_{1}^{\varepsilon}$ are finite, $\mathbb{E} e^{\beta Z_{1}^{\varepsilon}}=1$ for some $\beta=\beta(\varepsilon)>0$ and the Cramér estimate says that (see also Bertoin and Doney (1994))

$$
\mathbb{P}\left\{Z_{u}^{\varepsilon}>x \text { for some } u \geq 0\right\} \leq e^{-\beta x} .
$$

We also need a more accurate upper bound for $\mathbb{P}\left\{Z_{u}^{\varepsilon}>x\right.$ for some $\left.u \leq t\right\}$ for small values of $t$. Notice that, for all $s>0$, the process $e^{s\left(Z_{t}-\mathbb{E} Z_{t}\right)}$ is a positive submartingale, so Doob's inequality is applicable

$$
\begin{aligned}
\mathbb{P}\left\{Z_{u}^{\varepsilon}>x \text { for some } u \leq t\right\} & \leq \mathbb{P}\left\{Z_{u}-\mathbb{E} Z_{u}>x \text { for some } u \leq t\right\} \\
& \leq e^{-s x} \mathbb{E} e^{s\left(Z_{t}-\mathbb{E} Z_{t}\right)} \\
& =e^{-s x} e^{s^{2} t \sigma^{2} / 2} \mathbb{E} e^{s X_{t}^{(2)}}
\end{aligned}
$$

Recalling the upper bound (19) for $\mathbb{E} e^{s X_{t}^{(2)}}$, we get

$$
\mathbb{P}\left\{Z_{u}^{\varepsilon}>x \text { for some } u \leq t\right\} \leq e^{-s x} e^{\left(s^{2} \sigma^{2} / 2+c e^{s}\right) t} .
$$

For $t \leq e^{-1}$, taking $s:=\log \frac{1}{t}$ we finally get

$$
\mathbb{P}\left\{Z_{u}^{\varepsilon}>x \text { for some } u \leq t\right\} \leq c_{1} e^{-s x}=c_{1} t t^{x-1} \leq c_{1} t e^{1-x}=c_{2} t e^{-x}(24)
$$

Since $X_{1}$ is assumed to be strong subexponential, by Corollary 8 the distribution $\Pi(d x) / \Pi(1, \infty)$ concentrated on $(1, \infty)$ is strong subexponential too. Then the compound Poisson process $X_{t}^{\varepsilon}$ with intensity $\Pi(\mathbb{R} \backslash(-1,1))$ and drift $(a+\varepsilon) t$ satisfies all the conditions of Corollary 2 which implies, as $x \rightarrow \infty$ uniformly for all $t>0$,

$$
\begin{aligned}
\mathbb{P}\left\{X_{u}^{\varepsilon}>x \text { for some } u \leq t\right\} & \sim \frac{1}{|a+\varepsilon|} \int_{x}^{x+t|a+\varepsilon|} \bar{F}(v) d v \\
& \leq \frac{1}{|a+\varepsilon|} \int_{x}^{x+t|a|} \bar{F}(v) d v
\end{aligned}
$$


As follows from (23) and (24), uniformly for all $t>0$,

$\mathbb{P}\left\{Z_{u}^{\varepsilon}>x\right.$ for some $\left.u \leq t\right\}=o\left(\mathbb{P}\left\{X_{u}^{\varepsilon}>x\right.\right.$ for some $\left.\left.u \leq t\right\}\right) \quad$ as $x \rightarrow \infty$.

Since $X_{u}^{\varepsilon}$ and $Z_{u}^{\varepsilon}$ are independent, the last two relations allow us - following standard arguments for subexponential distributions - to conclude the following upper bound, uniformly for all $t>0$,

$$
\mathbb{P}\left\{X_{u}>x \text { for some } u \leq t\right\} \leq \frac{1+o(1)}{|a+\varepsilon|} \int_{x}^{x+t|a|} \bar{F}(v) d v \quad \text { as } x \rightarrow \infty .
$$

Letting $\varepsilon \downarrow 0$, we conclude the desired upper bound which together with the lower bound (21) implies the required asymptotics.

Similar to Theorem 4 we conclude with the following principle of a single big jump for the maximum $M_{t}$ of the Lévy process $X_{t}$. Let $T_{k}$ be the time

epoch of the $k$ th jump of the compound Poisson process $X_{t}^{(3)}$ with jump absolute values greater than 1 arising in the decomposition of $X_{t}$ into three independent processes. Let the events $D_{k}$ be defined literally in the same way as in Theorem 4, see (13).

Theorem 9. In conditions of Theorem 5, for any fixed $\varepsilon>0$,

$$
\lim _{A \rightarrow \infty} \lim _{t, x \rightarrow \infty} \mathbb{P}\left\{\cup_{k=1}^{N_{t}} D_{k} \mid M_{t}>x\right\} \geq \frac{|a|}{|a|+2 \varepsilon} .
$$

\section{Sampling of Lévy process}

The results presented in the last section allow us to derive useful in applications tail asymptotics for a Lévy process $X_{t}$ stopped at random time $\tau$ and for its maxima $M_{\tau}$ within this time interval.

Theorem 10. Assume that a positive random variable $\tau$ is independent of the Lévy process $X_{t}$. Let the distribution $F$ of $X_{1}$ be strong subexponential. If $a:=\mathbb{E} X_{1}<0$ then

$$
\mathbb{P}\left\{M_{\tau}>x\right\} \sim \frac{1}{|a|} \mathbb{E} \int_{x}^{x+\tau|a|} \bar{F}(y) d y \text { as } x \rightarrow \infty .
$$

Assume in addition that $\mathbb{E} \tau<\infty$. Then

(i) If $\mathbb{E} X_{1}<0$ then

$$
\mathbb{P}\left\{X_{\tau}>x\right\} \sim \mathbb{P}\left\{M_{\tau}>x\right\} \sim \mathbb{E} \tau \bar{F}(x) \text { as } x \rightarrow \infty
$$


(ii) If $\mathbb{E} X_{1} \geq 0$ and if there exists $c>\mathbb{E} X_{1}$ such that

$$
\mathbb{P}\{c \tau>x\}=o(\bar{F}(x)) \text { as } x \rightarrow \infty,
$$

then asymptotics (26) again hold.

A similar problem for Lévy processes with mixed-exponential jumps and for exponential $\tau$ was studied, e.g., by Mordecki [19], see also the references therein. Analogue results for random walks with subexponential jumps were proven in Denisov et al. (2010).

Proof. Conditioning on $\tau$ which is independent of $X_{t}$, we deduce that

$$
\mathbb{P}\left\{M_{\tau}>x\right\}=\int_{0}^{\infty} \mathbb{P}\left\{M_{t}>x\right\} \mathbb{P}\{\tau \in d t\} .
$$

Then by the uniform asymptotics for $M_{t}$ of Theorem 5 ,

$$
\mathbb{P}\left\{M_{\tau}>x\right\} \sim \frac{1}{|a|} \int_{0}^{\infty} \int_{x}^{x+t|a|} \bar{F}(v) d v \mathbb{P}\{\tau \in d t\} \quad \text { as } x \rightarrow \infty,
$$

and the first assertion (25) follows.

(i), (ii). Since $X_{\tau} \leq M_{\tau}$, it is sufficient to prove that

$$
\liminf _{x \rightarrow \infty} \frac{\mathbb{P}\left\{X_{\tau}>x\right\}}{\bar{F}(x)} \geq \int_{0}^{\infty} t \mathbb{P}\{\tau \in d t\}=\mathbb{E} \tau
$$

and

$$
\limsup _{x \rightarrow \infty} \frac{\mathbb{P}\left\{M_{\tau}>x\right\}}{\bar{F}(x)} \leq \mathbb{E} \tau \text {. }
$$

By subexponentiality of $X_{1}$, for all $t>0, \mathbb{P}\left\{X_{t}>x\right\} \sim t \bar{F}(x)$ as $x \rightarrow \infty$, regardless of the sign of $\mathbb{E} X_{1}$. Then conditioning on $\tau$ for $\mathbb{P}\left\{X_{\tau}>x\right\}$ implies (28) due to Fatou's lemma.

Let us now prove (29). If $\mathbb{E} X_{1}<0$ then (29) follows from (25) by the dominated convergence because

$$
\int_{x}^{x+|a| \tau} \bar{F}(v) d v \leq|a| \tau \bar{F}(x) .
$$

In the case $\mathbb{E} X_{1} \geq 0$, we start with the following upper bound: for all $N$,

$$
\begin{aligned}
& \mathbb{P}\left\{M_{\tau}>x\right\} \\
& \quad \leq \mathbb{P}\left\{M_{\tau}>x, \tau \leq N\right\}+\mathbb{P}\left\{M_{\tau}>x, \tau \in(N, x / c]\right\}+\mathbb{P}\{c \tau>x\} \\
& \quad=: P_{1}+P_{2}+P_{3} .
\end{aligned}
$$


By Theorem 5, for all $t, \mathbb{P}\left\{M_{t}>x\right\} \sim t \bar{F}(x)$ as $x \rightarrow \infty$. In addition, $M_{t} \leq M_{N}$ for $t \leq N$. Thus, the dominated convergence yields that, for any fixed $N$,

$$
\begin{aligned}
& \mathbb{P}\left\{M_{\tau}>x, \tau \leq N\right\} \\
& \quad=\int_{0}^{N} \mathbb{P}\left\{M_{t}>x\right\} \mathbb{P}\{\tau \in d t\} \sim \mathbb{E}\{\tau ; \tau \leq N\} \bar{F}(x) \text { as } x \rightarrow \infty
\end{aligned}
$$

In order to estimate $P_{2}$ we take $\varepsilon=\left(c-\mathbb{E} X_{1}\right) / 2>0$ and $b=\left(\mathbb{E} X_{1}+c\right) / 2$. Consider $\widetilde{X}_{t}:=X_{t}-b t$ and $\widetilde{M}_{t}=\sup _{u \leq t} \widetilde{X}_{u}$. Then $\mathbb{E} \widetilde{X}_{1}=-\varepsilon<0$ and Theorem 5 is applicable. Taking into account that $M_{t} \leq \widetilde{M}_{t}+b t$, we obtain that there exists $K$ such that, for all $x$ and $t$,

$$
\begin{aligned}
\mathbb{P}\left\{M_{t}>x\right\} & \leq \mathbb{P}\left\{\widetilde{M}_{t}>x-b t\right\} \\
& \leq K \int_{0}^{\varepsilon t} \overline{\widetilde{F}}(x-b t+y) d y \leq K \int_{0}^{\varepsilon t} \bar{F}(x-b t+y) d y .
\end{aligned}
$$

Hence,

$$
\begin{aligned}
P_{2}=\mathbb{P}\left\{M_{\tau}>x, \tau \in(N, x / c]\right\} & \leq K \int_{N}^{x / c} \mathbb{P}\{\tau \in d t\} \int_{0}^{\varepsilon t} \bar{F}(x-b t+y) d y \\
& =K \int_{N}^{x / c} \mathbb{P}\{\tau \in d t\} \int_{\mathbb{E} X_{1} t}^{b t} \bar{F}(x-y) d y
\end{aligned}
$$

because $b-\varepsilon=\mathbb{E} X_{1}$. Then

$$
\begin{aligned}
P_{2} & \leq K \int_{N \mathbb{E} X_{1}}^{b x / c} \bar{F}(x-y) d y \int_{\max (N, y / b)}^{x / c} \mathbb{P}\{\tau \in d t\} \\
& \leq K \int_{N \mathbb{E} X_{1}}^{b x / c} \bar{F}(x-y) \mathbb{P}\{\tau>y / b\} d y
\end{aligned}
$$

Owing $b<c$ and the condition (27), the inequality $\mathbb{P}\{\tau>y / b\} \leq K_{1} \bar{F}(y)$ holds for some $K_{1}$ and all $y$. Therefore,

$$
P_{2} \leq K K_{1} \int_{N \mathbb{E} \xi}^{b x / c} \bar{F}(x-y) \bar{F}(y) d y=o(\bar{F}(x)) \text { as } x, N \rightarrow \infty
$$

follows from $b / c<1$ and from $F \in \mathcal{S}^{*}$, see, e.g. Foss et al. (2013, Theorem $3.24)$.

Finally, by the condition $(27), P_{3}=o(\bar{F}(x))$ as $x \rightarrow \infty$. Substituting now (31) and (32) into (30) we conclude (29) and the proof is complete. 


\section{Application to ruin probabilities}

The results obtained above are directly applicable to the Cramér-Lundberg renewal model in the collective theory of risk defined as follows, see e.g. Asmussen and Albrecher (2010, Sec. X.3). We consider an insurance company and assume the constant inflow of premium occurs at rate $c$, that is, the premium income is assumed to be linear in time with rate $c$. Also assume that the claims incurred by the insurance company arrive according to a renewal process $N_{t}$ with intensity $\lambda$ and the sizes (amounts) $Y_{n} \geq 0$ of the claims are i.i.d. random variables with common distribution $B$ and mean $b$. The $Y_{n}$ 's are assumed to be independent of the process $N_{t}$. The company has an initial risk reserve $u=R_{0} \geq 0$. Then the risk reserve $R_{t}$ at time $t$ is equal to

$$
R_{t}=u+c t-\sum_{i=1}^{N_{t}} Y_{i}
$$

Then the probability

$$
\psi(u, t):=\mathbb{P}\left\{R_{s}<0 \text { for some } s \in[0, t]\right\}
$$

is the finite time horizon probability of ruin. The techniques developed for compound renewal process with drift provide a method for estimating the probability of ruin in the presence of heavy-tailed distribution for claim sizes. Since $c>0$, the ruin can only occur at a claim epoch. Therefore,

$$
\psi(u, t)=\mathbb{P}\left\{\sum_{i=1}^{n} Y_{i}-c T_{n}>u \text { for some } n \leq N_{t}\right\},
$$

where $T_{n}$ is the $n$th claim epoch. The last relation represents the ruin probability problem as the tail probability problem for the maximum of a compound renewal process with linear drift.

Let the net-profit condition $c>b \lambda$ hold, thus the process has a negative drift and $\psi(u, t) \rightarrow 0$ as $u \rightarrow \infty$ uniformly for all $t \geq 0$. Applying Theorem 1(i), we deduce the following result on the decreasing rate of the ruin probability to zero as the initial risk reserve becomes large in the case of heavy-tailed claim size distribution, compare with special cases of fixed $t$ in Asmussen and Albrecher (2010, Section X.4) and of the compound Poisson model in Foss et al. (2013, Theorem 5.21). 
Theorem 11. If the claim size distribution $B$ is strong subexponential, then

$$
\psi(u, t) \sim \frac{\lambda}{c-b \lambda} \int_{u}^{u+t(c / \lambda-b) \mathbb{E} N_{t}} \bar{B}(v) d v
$$

as $u \rightarrow \infty$ uniformly for all $t \geq 0$. In particular,

$$
\psi(u, t) \sim \frac{\lambda}{c-b \lambda} \int_{u}^{u+t(c-b \lambda)} \bar{B}(v) d v \quad \text { as } u, t \rightarrow \infty .
$$

Acknowledgment. The author is very thankful to two referees whose comments helped a lot to improve the paper.

[1] Asmussen, S., Applied Probability and Queues, 2nd edn. Springer, New York, 2003.

[2] Asmussen, S. and Albrecher, H., Ruin Probabilities, 2nd edn. World Scientific, Singapore, 2010.

[3] Asmussen, S., Subexponential asymptotics for stochastic processes: extremal behavior, stationary distributions and first passage probabilities. Annals Appl. Probab. 2 (1998) 354-374.

[4] Asmussen, S. and Klüppelberg, C., Large deviations results for subexponential tails, with applications to insurance risk. Stochastic Process. Appl. 64 (1996) 103-125.

[5] Asmussen, S., Schmidli, H., and Schmidt, V., Tail Probabilities for nonstandard risk and queueing processes with subexponential jumps. Adv. Appl. Prob. 31 (1999) 422-447.

[6] Bertoin, J. and Doney, R., Cramér's estimate for Lévy processes. Statist. Probab. Lett. 21 (1994) 363-365.

[7] Borovkov, A. A. and Borovkov, K. A., Asymptotic analysis of random walks. Heavy-tailed distributions, Cambridge Univ. Press, 2008.

[8] Braverman, M., Mikosch, T. and Samorodnitsky, G., Tail probabilities of subadditive functionals of Lévy processes. Ann. Appl. Probab. 12 (2002) 69-100.

[9] Denisov, D., Foss, S., and Korshunov, D., Asymptotics of randomly stopped sums in the presence of heavy tails. Bernoulli 16 (2010) 971994. 
[10] Doney, R., Klüppelberg, C. and Maller, R., Passage time and fluctuation calculations for subexponential Lévy processes. Bernoulli 22 (2016) $1491-1519$.

[11] Embrechts, P., Goldie, C., and Veraverbeke, N., Subexponentiality and infinite divisibility. Z. Wahrscheinlichkeitstheorie verw. Gebiete 49 (1979) 335-347.

[12] Embrechts, P., Klüppelberg, C., and Mikosch, T., Modelling Extremal Events for Insurance and Finance, Springer, Berlin, 1997.

[13] Foss, S., Konstantopoulos, T., and Zachary, S., Discrete and continuous time modulated random walks with heavy-tailed increments. J. Theor. Probab. 20 (2007) 581-612.

[14] Foss, S., Korshunov, D., and Zachary, S., An Introduction to HeavyTailed and Subexponential Distributions, 2nd Ed. Springer, New York, 2013.

[15] Klüppelberg, C., Kyprianou, A. E., and Maller, R. A., Ruin probabilities and overshoots for general Lévy insurance risk processes. Ann. Appl. Probab. 14 (2004) 1756-1801.

[16] Kyprianou, A. E., Introductory lectures on fluctuations of Lévy processes with applications. Springer, Berlin, 2006.

[17] Korshunov, D., On distribution tail of the maximum of a random walk. Stoch. Proc. Appl. 72 (1997) 97-103.

[18] Korshunov, D., Large-deviation probabilities for maxima of sums of independent random variables with negative mean and subexponential distribution. Theor. Probab. Appl. 46 (2002) 355-366.

[19] Mordecki, E., Ruin probabilities for Lévy processes with mixedexponential negative jumps. Theory Probab. Appl. 48 (2007) 170-176.

[20] Palmowski, Z. and Zwart, B., Tail asymptotics of the supremum of a regenerative process. J. Appl. Prob. 44 (2007) 349-365.

[21] Rhee, C.-H., Blanchet, J. and Zwart, B. Sample path large deviations for heavy-tailed Lévy processes and random walks. arXiv preprint arXiv:1606.02795 (2016).

[22] Rolski, T., Schmidli, H., Schmidt, V, and Teugels, J., Stochastic Processes for Insurance and Finance, Wiley, Chichester, 1998. 
[23] Sato, K., Lévy Processes and Infinitely Divisible Distributions, Cambridge Univ. Press, 1999.

[24] Willekens, E., On the supremum of an infinitely divisible process. Stoch. Proc. Appl. 26 (1987) 173-175. 